\title{
Realistic River Image Synthesis using Deep Generative Adversarial Networks
}

\author{
Akshat Gautam \\ Lambert High School \\ Suwanee, United States \\ akshatgautam@gmail.com \\ Muhammed Sit \\ Interdisciplinary Graduate Program in Informatic \\ University of Iowa \\ Iowa City, United States \\ muhammed-sit@uiowa.edu \\ Ibrahim Demir \\ Dept. of Civil \& Environmental Engineering \\ University of Iowa \\ Iowa City, United States \\ ibrahim-demir@uiowa.edu
}

\section{This article is a pre-print submitted to EarthArXiv.}

\begin{abstract}
In this paper, we investigate an application of image generation for river satellite imagery. Specifically, we propose a generative adversarial network (GAN) model capable of generating high-resolution and realistic river images that can be used to support models in surface water estimation, river meandering, wetland loss and other hydrological research studies. First, we summarized an augmented, diverse repository of overhead river images to be used in training. Second, we incorporate the Progressive Growing GAN (PGGAN), a network architecture that iteratively trains smaller-resolution GANs to gradually build up to a very high resolution, to generate $256 \times 256$ river satellite imagery. With conventional GAN architectures, difficulties soon arise in terms of exponential increase of training time and vanishing/exploding gradient issues, which the PGGAN implementation seems to significantly reduce. Our preliminary results show great promise in capturing the detail of river flow and green areas present in river satellite images that can be used for supporting hydroinformatics studies.
\end{abstract}

Index Terms-Generative Adversarial Networks (GANs), Satellite Imagery, Hydroinformatics, Deep Learning

\section{INTRODUCTION}

Hydroinformatics and remote sensing [1] make up two significant subfields of civil and environmental engineering. Both fields are used is conjunction for studying and mapping of water across the terrain, specifically looking at the flow of freshwater repositories like rivers and streams. These studies utilize satellite imagery in parallel with remote sensing and geographic information systems (GIS) [2] to better understand terrain, altitude, and contour mappings of rivers in the vicinity. This data can then be implemented to effectively model flood simulations, for example, to see which buildings and structures have the highest risk of being compromised within a floodprone area [3], [4]. Data can then be relayed to first responders in the area to maximize rescue efficiency and reduce any damage to life or property. As a result, it is often beneficial for engineers to have access to a large repository of satellite river images. Through this research study, we aim to create customshaped river images that can provide great detail to assist in flood prediction and hydrological modelling applications [5], [6].

Over time, rivers develop sinusoidal, curved features called meanders [7], due to the sideways flow of water and sediment that slowly erodes from the river banks. Because of their dynamic, ever-changing nature, they play a crucial role in the analysis of flood simulations and assessing the true scale of a river's surrounding area floodplain. The difficulty lies in analyzing a steady-state, long-term equilibrium that a river may converge to, since meandering rivers do not seem to have such an end behavior. As such, the curve and arc length of the meanders can be parametrized by non-linear and linear partial differential equations for fluid dynamics [8], [9]. To look at diverse examples of real-life meanderings in satellite imagery, it is of the utmost importance for hydrodynamics engineers to have a dataset that can provide them with thousands of visual samples. A real-time update of satellite view of major rivers can provide more and more optical clues about how these meanders change over time, even using the sequential aspects of a recurrent neural network and the image classification aspect of a convolutional neural network.

Deep generative learning refers to a subfield of artificial intelligence in which models learn to synthesize unique samples based on a given data distribution [10]. This falls under the category of unsupervised learning, where the task is datadriven and the model is not given any human input besides the dataset. For example, standard supervised learning algorithms can be comprised of Convolutional Neural Network (CNN) image classifiers, which analyze a pre-labeled set of thousands of images to find patterns between different annotated labels. Unsupervised generative learning, on the other hand, creates its own unique understanding of the dataset. Generative Adversarial Networks (GANs) [11] are the primary structure implemented for unsupervised data generation. Consisting of a generator and discriminator network, they work against each other in a minimax, zero sum game approach, where the former learns to generate realistic images capable of fooling the latter, which tries to differentiate between real and computersynthesized images. The generator takes a $\mathrm{Z}$ dimensional latent vector as input [11] (where $\mathrm{Z}$ is a vector somewhere in the Z-dimensional subspace called the latent space), and feeds this through a series of convolutional layers to generate an image tensor. The discriminator takes in an image tensor as input, and runs it through a series of convolutional layers and one sigmoid layer at the end, outputting a probability to discern whether the network's tensor input belongs to the original data 
distribution or not [10], [11].

Currently, GAN-based image synthesis yields very robust results when generating 64x64 images. By using other computational upsampling techniques like nearest neighbor and bilinear interpolation [12], while the output resolution does increase, a great amount of visual detail is lost, and the image becomes very blurry. Since such a standardized algorithm is applied evenly to every section of the image, a grid-like pattern seems to emerge among all of the images. On a standard computer screen, a $64 \times 64$ image is about 1 square inch. From an analytical perspective, this kind of image is quite small for the human eye to qualitatively differentiate rivers, terrain, and greenery. The actual detail of the image is limited by the amount of pixels present, which warrants the need for an exponentially higher resolution. Using a standard DCGAN [13], we generated 128x128 images and soon ran into an issue; the generator loss immediately zeroed out and the discriminator loss tended towards infinity. With each iteration, the loss remained the same and no parameters were updated. The images had a broad resemblance to satellite images, sharing a green background; however, any detail in the river shape or contour was not present. As a result, we utilize a Progressive Growing GAN [14] to generate high-resolution, 256x256 realistic river satellite imagery.

\section{RELATED WORK}

\section{A. Deep Learning Architectures}

The Deep Convolutional GAN (DCGAN) is the first major step taken in the field of GAN-guided image synthesis. It is a combination of a standard convolutional neural network and a feedforward neural network. Instead of working with pooling and hidden layers, the DCGAN replaces these with strided and upsampling layers for the generator, along with reverse transpose functions for the discriminator [13]. A latent vector $\mathrm{z}(1 \mathrm{x} 100)$ is given as input to the generator network, and then a series of convolution layers upsample the image to a $64 \times 64$ 3-channel RGB image. DCGAN has been trained on datasets like CIFAR-10 and LSUN, showing comparable advantages to techniques like K-means clustering [15], a completely supervised learning algorithm. Because of the DCGAN's ability to adeptly understand the latent space, Radford et. al note that the latent vector $\mathrm{z}$ can be modified to emphasize certain regions of an image [16]; for example, if training on face samples, the latent vector can be modified to force a picture with brown or black hair from the generator. The DCGAN does a good job of not mixing and matching parts from one image into another, so images show up with a sharp clarity on the output $64 \times 64$ resolution [13]. One limitation stands in the scalability of the data; going from generating $64 \times 64$ to $128 \times 128$ [14] or larger images requires at least a quadratic increase in the amount of data samples the model inputs, therefore requiring much higher computational power at the expense of potentially average results. Other GAN architectures, like the PGGAN [14] or StyleGAN [17], are capable of bypassing this training time-resolution tradeoff.
The CycleGAN model performs image-to-image translation on unpaired image datasets, learning to map a series of input images with some key object, to an output image; for example, the researchers developed a model that can turn a real landscape photograph into a painted portrait, given two datasets: landscapes and paintings [18], [19]. The primary reason of CycleGAN's applicability to this paper is in its high-resolution image synthesis; the model works with high input and yields high output image dimensions. In contrast to DCGAN's standard Binary Cross-Entropy loss [20], the CycleGAN utilizes a series of compounded cost functions depending upon the generator's ability to convert back and forth between the two image datasets; the function is shown to have a greater feature retention than a simple GAN.

Karras et al. propose a novel image generation architecture building upon their previous work regarding the Progressively Growing GAN (PGGAN) [14]. They propose an alternative technique refining the 'black-box' mystery hidden in the generator by removing its latent vector input and replacing it with a separately learned input parameter. Conventionally, most GAN architectures randomly sample a set of values to input as a tensor to the generator network. A separate 8-layer multilayer perceptron is used to create a non-linear mapping between the latent vector $\mathrm{w}$ and its corresponding latent space $\mathrm{W}$ [17]. Then, this mapping is introduced between layers, alternating with Gaussian noise. This approach focuses more on the refinable styles of each generated image, allowing for specifically altered vector inputs to generate varying kinds of images. For example, the Celeb-A dataset [21] is a series of celebrity face images that the StyleGAN was originally trained upon; the new architecture is capable of fine-tuning specific points in the image, like facial sharpness, nose size, jawline, hairline, etc. The LSUN dataset is a large repository of image datasets categorized by differing classes; the authors of the StyleGAN paper trained a model to generate bedrooms and could control the different colors and visual styles present in the room, like bed size, color, pillows, etc [17], [22]. Other applications to image generation include Stanford's Car dataset [23], which is comprised of thousands of pictures of automobiles and cars, all of which can be further refined and conditionally generated by the StyleGAN.

\section{B. Big Data in Hydroscience}

Beyond the concept of image generation and generative adversarial networks, machine learning and data analytics methods have a multitude of applications in hydroscience and predictive weather analytics. For example, LSTMs (long short-term memory networks) and RNNs [24]-[26] have been used to predict the chance of flooding based on daily water discharge and precipitation levels. Again LSTM networks are used to classify flood related tweets from unrelated ones during Hurricane Irma [27]. In other cases, feedforward deep neural networks have been used to improve the robustness of standard weather prediction methods; for example, by inputting infrared satellite imagery, microwave scans, and images from hundreds of satellites orbiting the Earth, scientists can train neural 
networks to predict the levels of snow over time [28] in areas more prone to map distortion, like at more extreme latitudes present in the North and South poles. Rather than just using one specific variable, like scans from a singular satellite or at a specific wavelength, deep learning enables scientists to consolidate numerous variables and use supervised neural networks and clustering algorithms (K-means) [29] to find novel trends. Other studies have looked at incorporating machine learning as an alternative to differential equations for modeling systems like water flow and runoff. For example, scientists have used polynomial and multivariate regression to analyze flow of a river with non-linear behavior over differing time intervals a day [30]. This enables them to individually analyze the value and contribution of each variable they record, towards the final prediction. Big data analytics applications in the field of hydroscience involves crowdsourced stage measurements [31], generating rainfall products from NEXRAD [32], intelligent systems for flooding [33], optimization of river network representation data models [34] and crowdsourced voluntary distribution of hydrologic model computations [35].

\section{Applications of GANs for Image Generation}

ArtGAN combines a DCGAN [13] architecture with a decoder-autoencoder system that gives the generator a noise vector input along with a specific label of art style, like impressionist, natural, abstract, etc. Unlike standard images like faces or cars, portraits are often open to massive amounts of interpretation that artists fill in. Using a dataset of precompiled artwork with different genres and labels, the authors input a randomized label corresponding to an art form, and then calculate the image loss based upon how strongly the image deviates from the rest of the training set images of that particular style [36]. Additionally, they note that the reconstruction aspect of decoding encoded images greatly improves the accuracy and efficiency of this hybrid GAN architecture, rather than having two separate, computationally expensive generator and discriminator networks in a minimax game. When downsampling to lower resolutions, the authors utilize overlapped average pooling layers [37], which keep the generator from using completely similar RGB pixel values across a large region of the image. To avoid a grid-like structure that usually shows up in high-resolution GANs due to upsampling layers, they used nearest neighbor upsampling [38].

To circumvent the issue of data scarcity, Wang et al. propose a CycleGAN implementation capable of taking computer graphics generated license plate images (random alphanumeric values superimposed on a colored rectangle), and converting them into photo-realistic license plate images that can then be used for image classification [39]. The trained CNN classifier is then given a few real images to further its accuracy, and can then be used in high-speed traffic cameras for real-time license plate capture and recognition. In place of a $\mathrm{CNN}$ discriminator classifying the generator's output as either real or fake, they incorporated PatchGANs [19], which are miniature discriminator networks that compute the realisticness of different $\mathrm{NxN}$ resolution image patches and then compute an average probability for the entire image. Isola et al. note that the most optimal value for $\mathrm{N}$ is 70 , with lower values producing very inaccurate results and higher values tending to overfit and generate excessive amounts of material in the image. Using techniques from the Wasserstein GAN, they propose a novel CycleGAN architecture with a better metric of measuring image and pixel-to-pixel distances to avoid gradient explosion and mode collapse [40].

Saadatnejad and Alahi develop a GAN architecture to synthesize pedestrian images in various poses on the street, which can then be used for self-driving car models to better discern pedestrians that can then be tracked and identified by the car's computer vision software [41]. The primary concern is the difficulty of tracking people in the absence or abundance of bad lighting, position changing, or occluding objects. For their generator, they used a pre-trained pose model and then mapped different pedestrian images to these poses, and then these images with their corresponding poses are inputted to a decoder that tries to integrate the pedestrians' images with a custom pose. This image is then analyzed by the discriminator. Instead of using a standard generator-discriminator layout, their generator is an encoder-decoder network [42] because of the extra 'pose' label that is required for all of their images. In the future, this encoder mixed with a generator could be used for conditional image generation, fusing a set of different labels (in this case, human poses) with standard images. Because of the similarity this task has with neural style transfer, the authors use style and perceptual losses to compare training dataset images with GAN-generated pedestrian poses.

\section{DATASET GENERATION}

\section{A. Data Collection}

Google Earth Engine is an online, interactive repository providing a full satellite mapping of the Earth, complete with all kinds of geological and hydrological landforms [43]. For this study, river images were captured using Google Earth Engine along different points spanning major rivers in the United States: Mississippi, Missouri, and Iowa River. 1,013 images were sampled evenly between these three rivers, and were captured via the ShareX screenshot editor in a native, 1000x1000 image resolution. Due to such a large initial image size, the dataset can then be cropped down accordingly depending on the type of GAN architecture that will be implemented. To provide a streamlined file formatting template, each image was saved in the format of: "[River Name]_[Year Taken]_[Image \#].jpg". Different rivers were used as sources of data so that the generative model will not stick to a specific kind of satellite background (eg. areas with high precipitation may have more lush, green imagery in the background, whereas more arid regions may have grass resembling a yellow or brown shade).

\section{B. Data Augmentation}

Data augmentation refers to the process of transforming the original dataset in such a way that the model is able to learn all 
kinds of variations, thereby improving its ability to generalize and become more cognizant of data samples that may differ from the statistical 'norm'. For this study, we applied 9 image transforms to the dataset, by employing Fast.AI's [44] vision.transforms computer vision library. These 9 transforms, applied to each image, created a total of 10 images per original in the dataset, thereby yielding 10,130 images in the final, augmented data distribution. The new file naming convention was: “[River Name]_[Year Taken]_[Original Image \#]_[Transform \#].jpg”. In Table I, a Python script was written utilizing Fast.AI's vision.transforms image processing library for data augmentation. The transforms mentioned above were applied to each of the original 1,013 images in the satellite river imagery dataset.

TABLE I

TRANSFORMS For DATA AUgMENTATION

\begin{tabular}{|c|}
\hline Transforms \\
\hline Random Cropping (2x) \\
\hline Hue/Saturation \\
\hline Dihedral (Flip + Rotate) \\
\hline Gaussian Noise \\
\hline Affine Transform \\
\hline Random Rotation (3x) \\
\hline
\end{tabular}

\section{Methodology}

\section{A. Progressively Growing GAN (PGGAN)}

When training the DCGAN [13] for 50 epochs, we obtained highly accurate and realistic image generation results; however, the DCGAN was only able to effectively generate 64x64 colored images, which is a very small resolution size. To be better analyzed by the human eye, we propose at least a $128 \times 128$ or $256 \times 256$ image size, which can lead to more qualitative observations. The CycleGAN [18] is used for unpaired image-to-image translation, so it can be used when trying to merge two different image datasets. In our study, we are trying to develop custom river satellite imagery of high resolutions, so the CycleGAN's translation aspect is not necessary. The StyleGAN [17] is a revolutionary GAN architecture that builds upon the PGGAN, improving its results and robustness by better manipulating the latent vector to style the generated images. However, the StyleGAN was primarily trained on datasets like Celeb-A [21], LSUN Bedrooms [22], or Stanford's Automobile dataset [23], all of which have specific objects that can vary in size and color when being generated. For example, Celeb-A's faces and LSUN's bedrooms can be modified using StyleGAN's new generator architecture to have different jawlines/hair color, or different pillows or wall colors, respectively [17]. With rivers, this style distinction is much more difficult to pinpoint, since each river has its own meanderings and concavities that cannot be visually modeled well [7]. As a result, we decided to use a Progressive GAN implementation to generate high-resolution, realistic satellite river imagery.

This paper provides a robust way to create high-resolution output images through a PGGAN network. The basic process is to train the discriminator at a very low resolution, initially starting at $4 \times 4$, and building the model up slowly and iteratively by adding layers and fine-tuning up to exponentially larger resolutions in powers of 2 [14]. The lower resolution images are prepared by performing a center crop on the input image to get to the desired input resolution. The adaptively growing nature of the networks makes it easier for them to learn different styles and components of an image; instead of having to learn how to just map a random noise latent vector to an image of massive resolution like $512 \times 512$ or $1024 \times 1024$, the networks learn gradually by starting with a simple $4 \times 4,8 \times 8,16 \times 16$, etc. At each resolution when a GAN is trained, there is a 'fadeout' block layer that helps to smoothen out the process of upscaling or downscaling between image dimensions. Another major improvement allowing PGGAN to do so well is through Wasserstein GAN Gradient Penalty (WGGAN-GP) loss [45]; the gradient is heavily penalized as the variations between synthesized images and the training dataset increase. This process allows the model to converge much faster throughout resolutions and still boast a better model accuracy via the Inception score [16], generating more realistic output images.

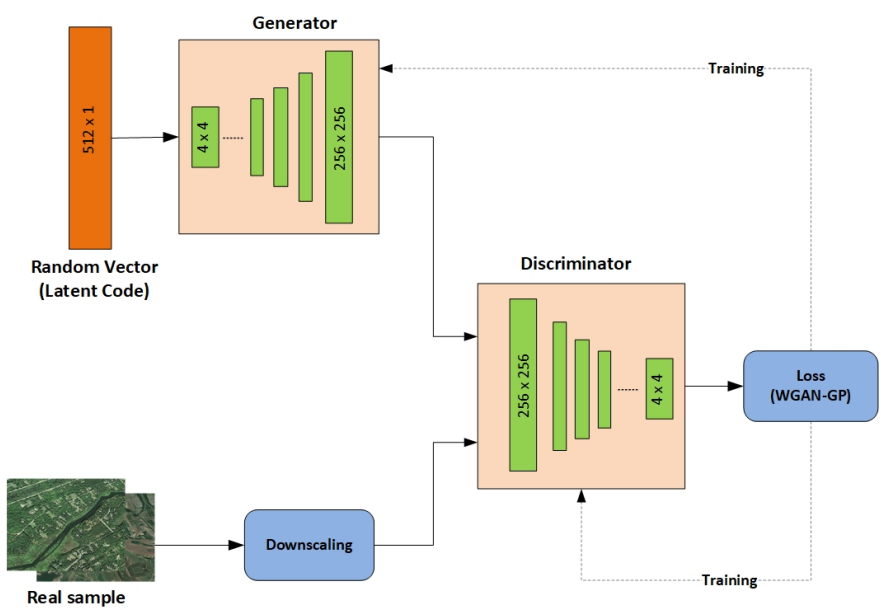

Fig. 1. PGGAN Architecture for Custom 256x256 Satellite Image Generation. Each tier/block of the image represents a resolution (power of 2) that the model is trained on. The generator is given a 512x1 latent vector input, and the discriminator is trained upon real samples to then discern the efficacy of the generator-created images. WGAN-GP loss can be pictured above for training both networks.

\section{B. Dataset Cropping}

From the custom river satellite imagery dataset, the images were originally at 1000x1000 resolution; however, when training on a PGGAN, a dataset must be cropped into progressively increasing resolutions in powers of 2. Usually, images are cropped down to the target resolution (e.g. 256x256 or $512 \times 512$ ), and then during training of each resolution, the images are center cropped down to smaller resolutions in real time. On the other hand, by pre-cropping all of the images into their specific folders, the GAN is able to devote all time and computational power towards training the generator and 
discriminator rather than doing random crops. We wrote a script to find the exact coordinate center of each river satellite image, and then perform a crop of a 256x256 grid in every cardinal direction. When first capturing the images in our dataset, we calibrated each of them so that the river or main water body was primarily located in the center of the image, which is why we decided to use a center crop rather than a random crop, which would end up adversely only taking greenery and excluding the actual rivers. In this case, the 10,130 images were each cropped into folders labeled '4', ' 8 ', . . ' 256', and due to the relatively small image resolution, this task is not very computationally extensive; 50,000 cropped images took up only $0.5 \mathrm{~GB}$ of space.

\section{Training Specifics}

The PGGAN model was trained for 73 hours on a Google Cloud Deep Learning Pytorch VM instance, on 2 NVIDIA Tesla V100 GPUs, with a total of 32 GB RAM, which allows us to use a larger batch size and latent vector space. We trained the PGGAN architecture with a Wasserstein GAN gradient penalty loss [45] and a batch size of 128. Because of the progressive architecture, we found it important to train even the smallest resolution images with meticulous training time and number of iterations; $4 \times 4$ and $8 \times 8$ images were trained for 48000 epochs, while image resolutions $16 \times 16$ to $256 \times 256$ were trained for 96000 epochs. The alpha (parameter for the intensity of fading/switching in between upscaled resolutions) started out at 1 , and then slowly decreased down to a value of approximately 0.537 at the end of training. The base learning rate was 0.001, and an Adam optimizer was used for training both the generator and discriminator networks (with beta1 and beta 2 being 0 and 0.99 , respectively). To improve the accuracy across increasing resolutions, we introduced 20,000 'fade-in' images between each upsampling $(4-8,8-16, \ldots)$.

Throughout the training session, the model kept checkpoints for every 16,000 iterations (for 7 resolutions, there were 42 checkpoints). After every checkpoint, a script was run to output the images generated during that specific training interval; across time, the images became greener and more realistic to mimic the diverse terrain in a satellite image and the model became very good at distinguishing the actual rivers from the green background. To view the metrics of the generator and discriminator loss functions, we used TensorboardX, and our visual observations soon matched the progressively improving trends found in the loss curves.

\section{Results}

This section underlines how PGGANs generate synthetic river satellite imagery. The results include instances of generated images, their evaluation using both computer vision and statistical losses, and their overall impact on hydroinformatics.

Figure 4 shows 64 samples of DCGAN synthesized satellite imagery, trained on a convolutional GAN for 50 epochs with a standard learning rate of 0.001 , and Adam optimizers for both the generator and discriminator networks. Although the rivers seem to be highly accurate and without many imperfections,

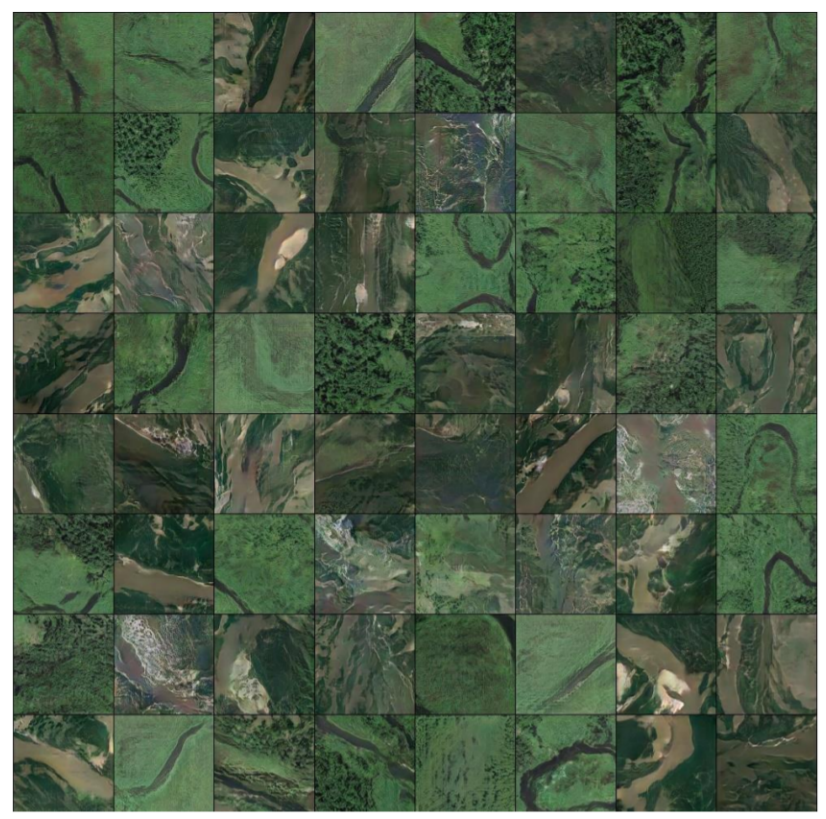

Fig. 2. Set of 256x256 GAN-Generated Satellite River Imagery. Across these 64 images, the final results show highly realistic river satellite imagery with little to no flaws in image quality. Specifically, this set of images was generated by the last checkpoint (iteration \#96,000 for the 256x256 generation block of the PGGAN).

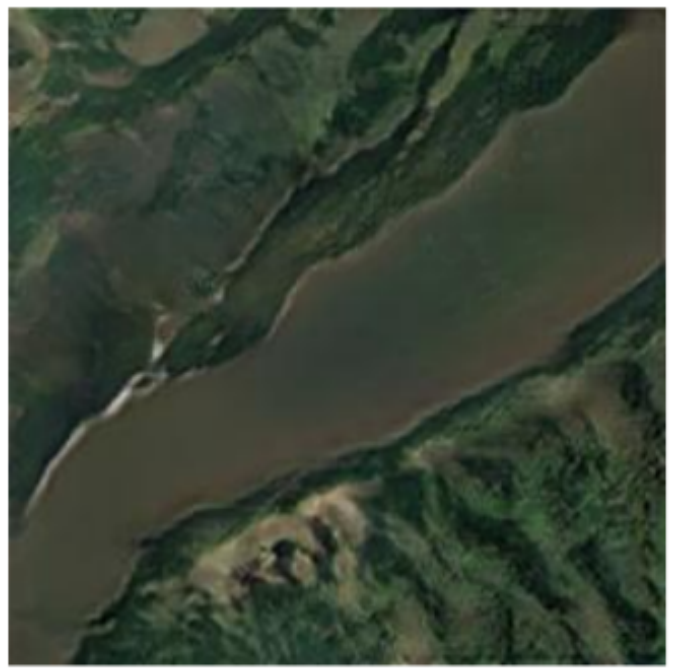

Fig. 3. 1 River Image (256x256 resolution). The river can clearly be seen running through the center of the image, and the PGGAN has done a good job of capturing the green detail present in the surrounding landscape. Additionally, there is no sign of any failure to figure out the color space of the image; even with thousands of images of rivers with a green background, the GAN is able to understand that this specific image should not have a green background, and therefore is able to adapt to the different styles present in the original training dataset. 

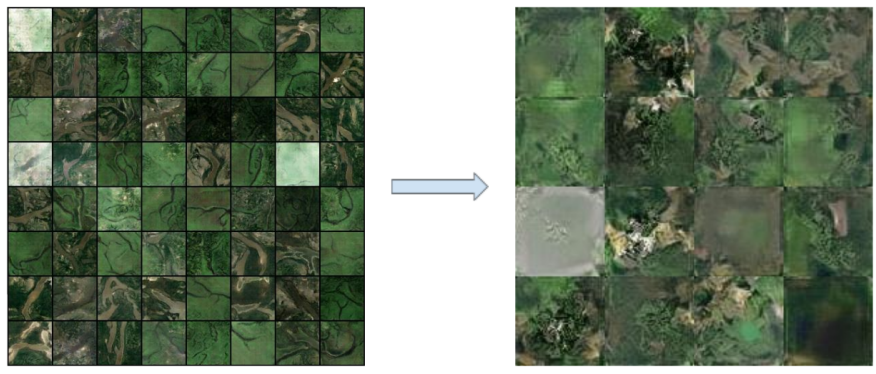

Fig. 4. The left side shows a sample of 64 DCGAN generated satellite images, of $64 \times 64$ resolution. The right side shows samples of DCGAN-generated images at $256 \times 256$ resolution; because of the DCGAN's inaccurate results on higher resolutions, we use the PGGAN network.

the images are of a very small size. Even with upsampling techniques like bilinear or cubic interpolation, the images will not be on par with a PGGAN-generated high-resolution sample. The image to the right shows our results when training a modified DCGAN with extra layers to produce $256 \times 256$ images. Even after 50 epochs, there are no signs of rivers forming in any of the images. This, compared with the results from our PGGAN implementation, further reinforces the benefits we found when generating high-resolution satellite imagery.

\section{A. Laplacian SWD}

SWD, or Sliced Wasserstein Distance, is a metric that measures the overall deviation between the original training dataset and the generator-synthesized dataset [46]. The standard Wasserstein Distance is difficult to compute on such high-dimensional input, especially in images due to the 3color channel RGB values attached to each tensor. Each image is turned into a Laplacian pyramid [46], a multi-scaled data structure with layers representing each resolution that the image was generated at. To cross resolutions, there are upsampling, downsampling, and blurring functions applied to the original large image, which are then all compiled into one file. The Laplacian SWD metric converts high-dimensional inputs into one-dimensional distributions, which are randomly sampled across the image and then added up as a loss function. We computed the Laplacian SWD [46], [47] score for key resolutions across the PGGAN's training (specifically resolutions $32 \rightarrow 256$ ). As seen in Table II, these normalized values tended to significantly decrease over time as the resolutions grew. Our PGGAN's accuracy actually increased with image size, unlike conventional GAN techniques that tend to have bouts of vanishing/exploding gradient when training upon exponentially higher image sizes.

\section{B. Inception Score}

The inception score (IS) refers to a metric for measuring the validation and accuracy of image generation GANs. Using a pre-trained CNN Inception v3 image classifier [48], the algorithm classifies and categorizes the generated images into various different classes based upon the diversity and image quality of the synthesized dataset [16]. The optimal value for
TABLE II

LAPLACIAN SWD ACROSS IMAGE RESOLUTIONS

\begin{tabular}{|l|l|l|l|l|}
\hline Resolution & 32 & 64 & 128 & 256 \\
\hline Laplacian SWD Score & 0.01337 & 0.0039 & 0.00322 & 0.00529 \\
\hline
\end{tabular}

The sliced Wasserstein distance significantly decreased across increasing resolutions; this may be attributed to the PGGAN's ability to learn weights gradually across smaller resolutions and then fine-tune them when building up to image sizes greater than $64 \times 64$.

an inception score is generally the amount of classes present within the data. Since our data was not labeled, it becomes slightly more difficult to discern what this maximum optimal value should be. We sampled information from 3 different rivers: the Mississippi, Missouri, and Iowa rivers. Below, we see the diversity of data presented by each river, with different background greenery, river shape, and overall terrain. As such, we can qualitatively discern that our maximum Inception score is equal to the number of labels/classes present: 3 .

Our inception value for the river image dataset was approximately 2.80548 . With approximately 3 main classes present in our data, we can conclude that our PGGAN implementation for synthesizing custom river satellite imagery is quite efficient and accurate.

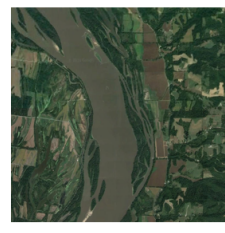

Class 1

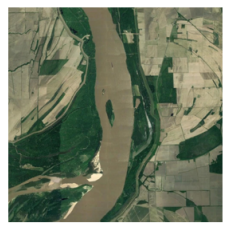

Class 2

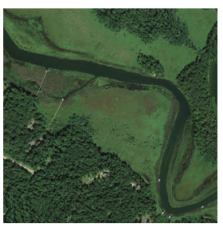

Class 3
Fig. 5. These seem to be the qualitatively differentiated classes present in the original dataset of 10,130 satellite river images. They all have different colored backgrounds comprising different kinds of greenery and bushes, and the rivers themselves each have a different color.

\section{CONCLUSION}

The preliminary results in our study show how PGGANs can be implemented to generate custom, 256x256 realistic river satellite imagery with the help of dataset augmentation. This paper demonstrates a novel dataset for hydroinformatics and remote sensing with its repository and augmentation of thousands of overhead river satellite images. In the future, other kinds of GANs can be incorporated to improve the customizability and applicability of these river images; for example, the CycleGAN and other image-to-image translation techniques [18], [19] can be utilized to translate a set of drawings/sketches of curves into custom shaped rivers, which can be used by hydroinformatics researchers to develop different kinds of synthetic river images for varying simulations.

The models utilized in this study can be further trained on higher resolutions to improve the efficacy of these satellite images towards different kinds of machine learning tasks; a GAN-based data augmentation platform would be very beneficial for training CNN image classifiers on satellite and terrain 
imagery to detect any changes in greenery, precipitation, or other GIS information. Overall, the results in our study show that PGGANs are very good at analyzing set of diverse terrain datasets (satellite images have varying backgrounds, river shapes, and greenery), and can quickly learn to model such data distributions to generate realistic river satellite imagery.

\section{ACKNOWLEDGMENT}

The work reported in this project has been possible with the support and work of many members of the Iowa Flood Center at the IIHR Hydroscience and Engineering, University of Iowa.

\section{REFERENCES}

[1] Y. Chen and D. Han, "Big data and hydroinformatics," Journal of Hydroinformatics, vol. 18, no. 4, pp. 599-614, 2016.

[2] J.-Y. Choi, B. A. Engel, and R. L. Farnsworth, "Web-based gis and spatial decision support system for watershed management," Journal of Hydroinformatics, vol. 7, no. 3, pp. 165-174, 2005.

[3] E. Yildirim and I. Demir, "An integrated web framework for hazus-mh flood loss estimation analysis," Natural Hazards, vol. 99, no. 1, pp. 275-286, 2019.

[4] L. J. Weber, M. Muste, A. A. Bradley, A. A. Amado, I. Demir, C. W. Drake, W. F. Krajewski, T. J. Loeser, M. S. Politano, B. R. Shea et al., "The iowa watersheds project: Iowa's prototype for engaging communities and professionals in watershed hazard mitigation," International journal of river basin management, vol. 16, no. 3, pp. 315-328, 2018.

[5] I. Yucel, A. Onen, K. Yilmaz, and D. Gochis, "Calibration and evaluation of a flood forecasting system: Utility of numerical weather prediction model, data assimilation and satellite-based rainfall," Journal of Hydrology, vol. 523, pp. 49-66, 2015.

[6] I. Demir, E. Yildirim, Y. Sermet, and M. A. Sit, "Floodss: Iowa flood information system as a generalized flood cyberinfrastructure," International journal of river basin management, vol. 16, no. 3, pp. 393-400, 2018.

[7] J. M. Hooke, "Complexity, self-organisation and variation in behaviour in meandering rivers," Geomorphology, vol. 91, no. 3-4, pp. 236-258, 2007.

[8] C. Camporeale, P. Perona, A. Porporato, and L. Ridolfi, "On the longterm behavior of meandering rivers," Water resources research, vol. 41, no. 12, 2005.

[9] R. C. Brower, D. A. Kessler, J. Koplik, and H. Levine, "Geometrical models of interface evolution," Physical Review A, vol. 29, no. 3, p. 1335, 1984.

[10] M. Ranzato, J. Susskind, V. Mnih, and G. Hinton, "On deep generative models with applications to recognition," in CVPR 2011. IEEE, 2011, pp. 2857-2864.

[11] I. Goodfellow, J. Pouget-Abadie, M. Mirza, B. Xu, D. Warde-Farley, S. Ozair, A. Courville, and Y. Bengio, "Generative adversarial nets," in Advances in neural information processing systems, 2014, pp. 2672 2680.

[12] R. Olivier and C. Hanqiang, "Nearest neighbor value interpolation," Int. J. Adv. Comput. Sci. Appl, vol. 3, no. 4, pp. 25-30, 2012.

[13] A. Radford, L. Metz, and S. Chintala, "Unsupervised representation learning with deep convolutional generative adversarial networks," arXiv preprint arXiv:1511.06434, 2015.

[14] T. Karras, T. Aila, S. Laine, and J. Lehtinen, "Progressive growing of gans for improved quality, stability, and variation," arXiv preprint arXiv:1710.10196, 2017.

[15] S. Lloyd, "Least squares quantization in pcm," IEEE transactions on information theory, vol. 28, no. 2, pp. 129-137, 1982.

[16] T. Salimans, I. Goodfellow, W. Zaremba, V. Cheung, A. Radford, and $\mathrm{X}$. Chen, "Improved techniques for training gans," in Advances in neural information processing systems, 2016, pp. 2234-2242.

[17] T. Karras, S. Laine, and T. Aila, "A style-based generator architecture for generative adversarial networks," in Proceedings of the IEEE Conference on Computer Vision and Pattern Recognition, 2019, pp. 4401-4410.
[18] J.-Y. Zhu, T. Park, P. Isola, and A. A. Efros, "Unpaired image-to-image translation using cycle-consistent adversarial networks," in Proceedings of the IEEE international conference on computer vision, 2017, pp. 2223-2232.

[19] P. Isola, J.-Y. Zhu, T. Zhou, and A. A. Efros, "Image-to-image translation with conditional adversarial networks," in Proceedings of the IEEE conference on computer vision and pattern recognition, 2017, pp. 11251134.

[20] Z. Zhang and M. Sabuncu, "Generalized cross entropy loss for training deep neural networks with noisy labels," in Advances in neural information processing systems, 2018, pp. 8778-8788.

[21] Z. Liu, P. Luo, X. Wang, and X. Tang, "Large-scale celebfaces attributes (celeba) dataset," Retrieved August, vol. 15, p. 2018, 2018.

[22] F. Yu, A. Seff, Y. Zhang, S. Song, T. Funkhouser, and J. Xiao, "Lsun: Construction of a large-scale image dataset using deep learning with humans in the loop," arXiv preprint arXiv:1506.03365, 2015.

[23] J. Krause, M. Stark, J. Deng, and L. Fei-Fei, "3d object representations for fine-grained categorization," in Proceedings of the IEEE international conference on computer vision workshops, 2013, pp. 554-561.

[24] G. Tang, D. Long, A. Behrangi, C. Wang, and Y. Hong, "Exploring deep neural networks to retrieve rain and snow in high latitudes using multisensor and reanalysis data," Water Resources Research, vol. 54 , no. 10 , pp. 8253-8278, 2018.

[25] M. Sit and I. Demir, "Decentralized flood forecasting using deep neural networks," arXiv preprint arXiv:1902.02308, 2019.

[26] Z. Xiang, J. Yan, and I. Demir, "A rainfall-runoff model with 1stm-based sequence-to-sequence learning," Water resources research, vol. 56, no. 1 , 2020.

[27] M. A. Sit, C. Koylu, and I. Demir, "Identifying disaster-related tweets and their semantic, spatial and temporal context using deep learning, natural language processing and spatial analysis: a case study of hurricane irma," International Journal of Digital Earth, vol. 12, no. 11, pp. 1205-1229, 2019. [Online]. Available: https://doi.org/10.1080/17538947.2018.1563219

[28] X.-H. Le, H. V. Ho, G. Lee, and S. Jung, "Application of long short-term memory (lstm) neural network for flood forecasting," Water, vol. 11, no. 7, p. $1387,2019$.

[29] R. Tse, "Towards general semi-supervised clustering using a cognitive reinforcement k-iteration fast learning artificial neural network ( $\mathrm{r}$ kflann)," Ph.D. dissertation, 2010.

[30] L. See, D. Solomatine, R. Abrahart, and E. Toth, "Hydroinformatics: computational intelligence and technological developments in water science applications," Hydrological Sciences Journal, vol. 52, no. 3, pp. 391-396, 2007

[31] Y. Sermet, P. Villanueva, M. A. Sit, and I. Demir, "Crowdsourced approaches for stage measurements at ungauged locations using smartphones," Hydrological Sciences Journal, pp. 1-10, 2019.

[32] B.-C. Seo, M. Keem, R. Hammond, I. Demir, and W. F. Krajewski, "A pilot infrastructure for searching rainfall metadata and generating rainfall product using the big data of nexrad," Environmental modelling \& software, vol. 117, pp. 69-75, 2019.

[33] Y. Sermet and I. Demir, "An intelligent system on knowledge generation and communication about flooding," Environmental modelling \& software, vol. 108, pp. 51-60, 2018.

[34] I. Demir and R. Szczepanek, "Optimization of river network representation data models for web-based systems," Earth and Space Science, vol. 4, no. 6, pp. 336-347, 2017.

[35] R. Agliamzanov, M. A. Sit, and I. Demir, "Hydrology@ home: a distributed volunteer computing framework for hydrological research and applications," Journal of Hydroinformatics, 2017.

[36] W. R. Tan, C. S. Chan, H. E. Aguirre, and K. Tanaka, "Artgan: Artwork synthesis with conditional categorical gans," in 2017 IEEE International Conference on Image Processing (ICIP). IEEE, 2017, pp. 3760-3764.

[37] D. Scherer, A. Müller, and S. Behnke, "Evaluation of pooling operations in convolutional architectures for object recognition," in International conference on artificial neural networks. Springer, 2010, pp. 92-101.

[38] A. Odena, V. Dumoulin, and C. Olah, "Deconvolution and checkerboard artifacts," Distill, vol. 1, no. 10, p. e3, 2016.

[39] X. Wang, Z. Man, M. You, and C. Shen, "Adversarial generation of training examples: applications to moving vehicle license plate recognition," arXiv preprint arXiv:1707.03124, 2017.

[40] M. Arjovsky, S. Chintala, and L. Bottou, "Wasserstein gan," arXiv preprint arXiv:1701.07875, 2017 
[41] S. Saadatnejad and A. Alahi, "Pedestrian image generation for selfdriving cars," Tech. Rep., 2019.

[42] Y. Pu, Z. Gan, R. Henao, X. Yuan, C. Li, A. Stevens, and L. Carin, "Variational autoencoder for deep learning of images, labels and captions," in Advances in neural information processing systems, 2016, pp. 2352-2360.

[43] N. Gorelick, M. Hancher, M. Dixon, S. Ilyushchenko, D. Thau, and R. Moore, "Google earth engine: Planetary-scale geospatial analysis for everyone," Remote sensing of Environment, vol. 202, pp. 18-27, 2017.

[44] J. Howard et al., "fastai," https://github.com/fastai/fastai, 2018.

[45] I. Gulrajani, F. Ahmed, M. Arjovsky, V. Dumoulin, and A. C. Courville, "Improved training of wasserstein gans," in Advances in neural information processing systems, 2017, pp. 5767-5777.

[46] I. Deshpande, Z. Zhang, and A. G. Schwing, "Generative modeling using the sliced wasserstein distance," in Proceedings of the IEEE conference on computer vision and pattern recognition, 2018, pp. 3483-3491.

[47] K. Shmelkov, C. Schmid, and K. Alahari, "How good is my gan?" in Proceedings of the European Conference on Computer Vision (ECCV), 2018, pp. 213-229.

[48] C. Szegedy, V. Vanhoucke, S. Ioffe, J. Shlens, and Z. Wojna, "Rethinking the inception architecture for computer vision," in Proceedings of the IEEE conference on computer vision and pattern recognition, 2016, pp. $2818-2826$. 